\title{
Monitoring Cells and Spores by Biomimetic Sensors - Applications in Biotechnology and Biological Threat
}

\author{
Franz L. Dickert ${ }^{1}$, Serpil Can ${ }^{1}$, Renata Samardzic ${ }^{1}$, Hermann F. Sussitz ${ }^{1}$, Usman Latif ${ }^{2}$ \\ ${ }^{1}$ University of Vienna, Department of Analytical Chemistry, Waehringer Str. 38, A-1090 Wien, Austria, \\ Franz.Dickert@univie.ac.at \\ ${ }^{2}$ COMSATS Institute of Information Technology, Abbottabad, Pakistan
}

\begin{abstract}
:
Biological species such as cells are permanently changing their properties due to environmental conditions and nutrition. An innovative recognition strategy is based on patterning polymers with bioparticles of interest. Thus, cavities are generated which engulf e.g. cells and spores. An efficient enrichment of analytes on the surface of these coatings can be detected by QCMs (Quartz-CrystalMicrobalances). The mass-sensitive QCM data were compared with AFM (Atomic-Force-Microscopy) measurements, where e.g. Escherichia coli bacteria adhered on a sensor coating were directly counted. Furthermore, the conversion of spores to bacteria can be monitored when sufficient nutrition is guaranteed. This process was studied via B. subtilis spores / bacteria as model for anthrax threat. Both the decay of spores and the growth of bacteria can be monitored by a Sauerbrey effect via a frequency alteration. The bacteria are selectively incorporated into the sensor layer which consists of a cross linked polyurethane. A glucose concentration of $10 \%$ in solution yields saturation effects concerning the influence of nutrition. Furthermore, progress was achieved in detecting cells by patterning with plastic cells which were generated by double imprinting by native analogues. Thus, sensor coatings can be reproduced easily. Synchronized cells can be used for selective monitoring of cells at a distinct growing phase. Thus, the type of cells, e.g. the bacterium strain, stages of cell reduplication and extent of nutrition can be recognized by biomimetic sensor strategies.
\end{abstract}

Key words: monitoring cells and spores, imprinting, QCM sensors, AFM

\section{Introduction}

Cells are permanently changing their properties e.g. size, because their metabolism depends on environmental conditions as well as nutrition. Characterization of these biological species can be performed by physical methods such as laser light scattering or biological procedures according to DNA analysis. In above mentioned assays, either selectivity is to be compromised or the time efforts are too long for rapid analysis. The online biosensor can be realized by characterizing the size and shape of bacteria cells, AFM image of these is shown in Fig. 1. Furthermore, the cell surface is also very important in respect to its chemical composition. Biomimetic recognition is an excellent platform to perform these tasks [1]. This strategy can be realized by surface imprinting as shown in Fig. 2. For this purpose, the cells of the bacteria or other bioparticles are assembled on a stamp. This arrangement is used for patterning a pre-polymer. The monomers such as $p, p^{\prime}$-isocyanatodiphenyl-methane, bisphenolA, poly-4-vinylphenol and phlororoglucinol as crosslinker form a gel at elevated temperatures. This pre-polymer is diluted by tetrahydrofuran and is applied to the QCM by a spin-spin off process. The sensitive electrode is patterned by a stamp with assembled bio-analytes. After

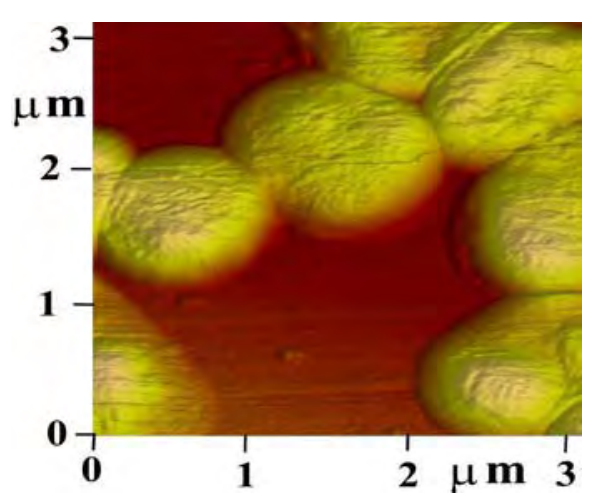

Fig. 1. Assembled Escherichia coli bacteria characterized by AFM.

cross-linking over night the stamp is removed. The bioparticles are washed out after the surface imprinting process. The reference electrode for compensating physical interferences as temperature, pressure fluctuations and un- 
selective adsorption is coated with the same, however, non-imprinted polymer.

The Fig. 2 exhibits how patterning process creates hollows on surfaces for size selective incorporation of cells and spores. An effective

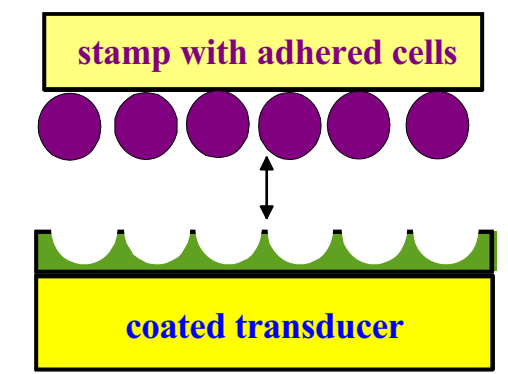

Fig. 2. Transducer (e.g. QCM) coated with a pre-polymer followed by surface patterning by a stamp with adhered cells

inclusion is guaranteed if the bio-particle fits into the hollow on the surface. Very large analytes cannot penetrate into the cavity, whereas, for small particles only a minor interaction with analyte and sensor coating is observed. Thus, it can be concluded that size is an exclusion principle which will not necessarily lead to a strong binding between the analyte and sensor coating. When the analyte is geometrically adapted to the hollow a strong intermolecular interaction between them will result, however, in a strong binding due to enthalpic contributions. Thus, the polarity of the coating surface must show strong affinity to the boundary of the bio-particle. Entropy contributions are of minor importance, if the binding of the particle is not favored, since it will gain more degrees of freedom in solution than bound to a sensor layer.

In this way a large variety of analytes in $\mathrm{nm}$ to $\mu \mathrm{m}$ dimension can be chemically recognized. Mass-sensitive transducers are very versatile detectors for this purpose. This strategy allows selective "on-line" monitoring of bio-particles directly in solution as in bioreactors. Selectivity makes it possible to distinguish even different strains of bacteria and their respective spores. Thus, the conversion of spores to cells can be followed as function of nutrition. Furthermore, improvements are obtained by imprinting with plastic bio-particles which enhance the robustness of the procedure described.

\section{Sensor Characteristics - Comparison of QCM with AFM Measurements}

The dual electrode QCM measurements for detecting e.g. Escherichia coli bacteria were performed in a flow cell. One electrode of the QCM was coated with bacteria-imprinted highly cross-linked polyurethane, whereas, the other one was non-imprinted. In each case, the reversibility of cell adhesion and dissociation was checked. The time constants of these processes was in the range of some minutes. The differences between both signals will compensate for non-selective responses. In Fig. 3. the sensor characteristic for Escherichia coli bacteria is shown in aqueous solution. A linear relation can be observed in this concentration range. Thus, not all available receptor sites on the surface are occupied by the analytes under this condition. Otherwise, saturation effects

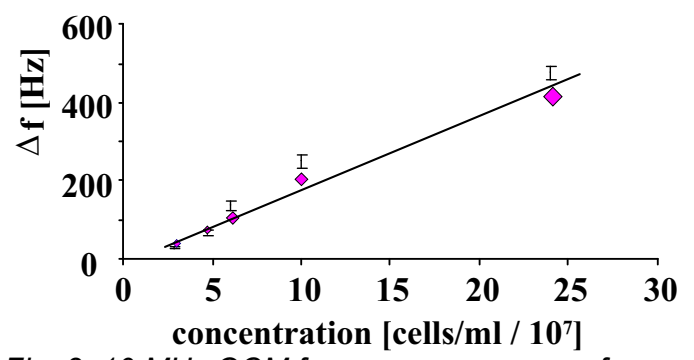

Fig. 3. $10 \mathrm{MHz} Q C M$ frequency response of Escherichia coli bacteria as function of cell concentrations ( $\diamond)$, compared to AFM-

measurements (I) by counting adhered cells

would be observed which flatten off the isotherm of adsorption at higher concentration. The frequency responses are directly related to the mass of the surface adhered bio-analytes. In this way these measurements can directly be compared with AFM measurements.

For doing this, the QCM surface must be conditioned as in case of mass-sensitive measurements. After patterning the sensor layers they were washed to remove the bacteria, followed by a equilibration to water and a distinct concentration of bacteria. Approximately, equilibrium according to cell incorporation is obtained after ten minutes. A short washing step of one second removes the non-selectively adhered bacteria. After evaporating solvent, the incorporated bacteria can be counted using the contact mode of AFM. The volumes of the bacteria solutions and their concentrations have to be adjusted, respectively to avoid multilayer adsorption. Otherwise, the first layer could not unambiguously detected, since it is covered by overlying cells. The AFM and QCM data can be related by the density of the bacteria which is near to $1 \mathrm{~g} / \mathrm{mL}$. A frequency response of 1000 $\mathrm{Hz}$ corresponds to a mass of approximately one micro-gram. Thus, the AFM counting procedure and frequency measurements with QCMs can directly be correlated. The satisfying accordance of these different measuring principles are shown in Fig. 3. Obviously, the bacteria are tightly bound to the patterned sensor surface. Both layer and adhered analytes show a behavior of a rigid solid which leads to a Sauerbrey [2] response - frequency 
diminishing effect. The linear sensor characteristic indicates a strong binding of bacteria to receptor sites generated by patterning polyurethane layers. Washing the sensor coating with adhered bacteria allows to differentiate between the analytes bound to cavities and those to less favorable interstitial sites.

\section{Detection of Spores}

Threat to mankind by bacteria is obvious. In this case, bacillus thuringiensis and bacillus subtilis bacteria were studied as model system for the dangerous anthrax bacillus. Bacillus thuringiensis strains produce proteins which act as pesticides against insects. These cells belong to the procaryotes, which lack a cell nucleus. They are aerobic and form as other bacillus types, the endospores. Insufficient nutrition will lead to spores which show a high robustness to temperature, dryness and deficiency of nutrients. The spores of bacillus anthracis are important according to their high potential threat in bio-terrorism. The spores of bacteria subtilis and thuringiensis can be used as model system to detect anthrax spores. For this purpose, highly cross-linked polyurethane coated 10 $\mathrm{MHz}$ QCMs were patterned with spores as analyte of interest to follow frequency responses. In Fig. 4, the AFM image of bacillus thuringiensis spores assembled on a flat surface is shown.

In Fig. 5 the $10 \mathrm{MHz}$ QCM frequency response to bacteria subtilis spores can be seen. For this purpose the spores were assembled on a stamp and pressed in a pre-polymer. The sensor device can be used after curing and

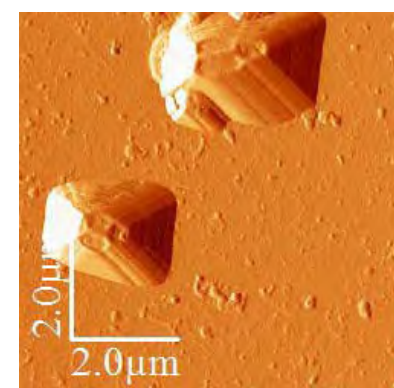

Fig. 4. AFM - microscopy of Bacillus thuringiensis spores adhered to a flat surface

washing off the template. Again, an imprinted and a non-imprinted polyurethane coating on a dual electrode QCM sensor was applied. An appreciable difference between both responses is obtained reflecting the selective receptor sites on imprinted materials (Fig. 5). A high reversibility was achieved to guarantee rapid sensing of bioanalytes. In the concentration range of $10^{6}$ to $10^{7}$ cells / $\mathrm{mL}$ a nearly linear behavior is found within two orders of magnitude which shows the absence of sensor saturation.

In Fig. 6 the cross sensitivities between spores of bacillus thuringiensis and subtilis can be seen. The sensor response shows the highest frequency shift only if the template is identical to the analyte. Minor responses are observed for

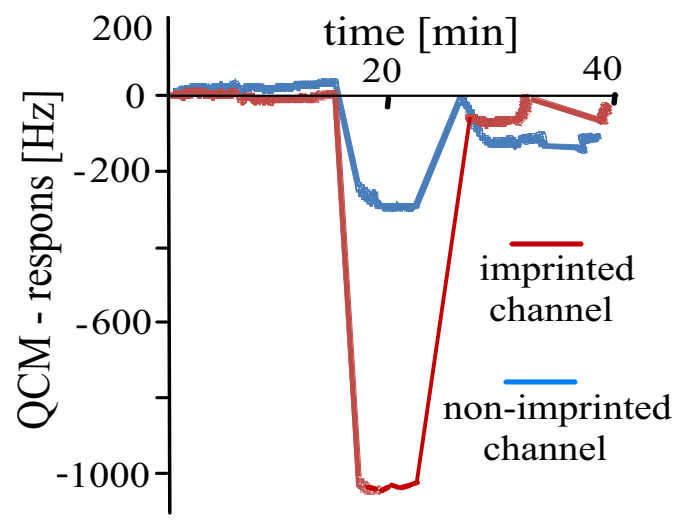

Fig. 5. $10 \mathrm{MHz}$ QCM frequency responses to bacteria subtilis spores to imprinted (--) and non-imprinted (--) dual electrodes as function of time response and recovery behavior

cross-sensitivities. These results are not based on differences in size and shape of the spores,

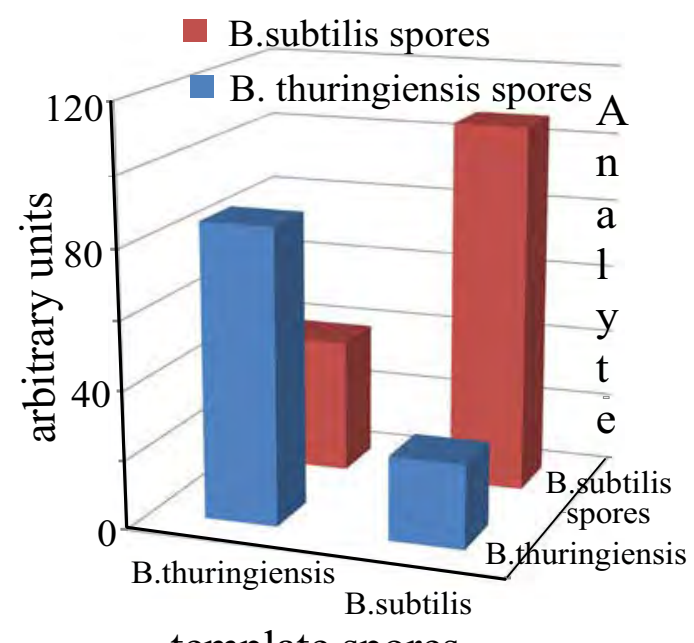

template spores

Fig. 6. Imprinting with B. subtilis and B. thuringiensis spores, QCM frequency responses, cross-sensitivities

since they have similar dimensions in the range of $3-4 \mu \mathrm{m}$. The selective frequency responses observed are primarily due to surface properties of the bio-particles. Thus, the polymer for the imprinting process must be optimized to achieve a strong interaction between the receptor sites and bio-particles. A straightforward strategy for successful imprinting can be realized by varying the phenolic ingredient of the polymer. This leads to a suitable polarity of the 
polymer and its extent of forming hydrogen bonds.

\section{Biotechnology - Monitoring bacterial Growth in Nutrient Solutions by QCM- Sensors}

Bacteria are formed in nutrient solutions from the respective spores. Important ingredients are e.g. glucose, hydrolysed proteins, ammonium sulfate, phosphates and other inorganic salts. The transformation of subtilis spores to bacteria is shown in Fig. 7 by light microscopy. Bacillus

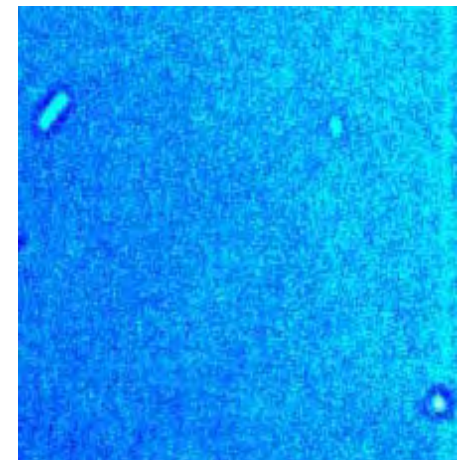

Fig. 7. Light microscopy, formation of bacillus subtilis from its spores in nutrient solution

subtilis is formed after an incubation period of approximately 6 hours. This process and the growing of bacteria can be monitored as function of time with a QCM-sensor as shown in Fig. 8. The sensor coating was printed with bacillus subtilis which guarantees a selective incorporation of this analyte. This is especially

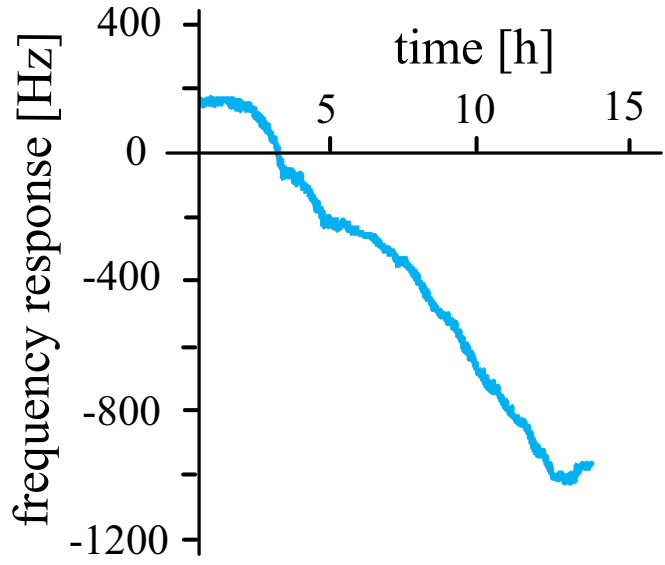

Fig. 8. Monitoring of bacillus subtilis from its spores in nutrient solution (2\% Ammoniumsulfate, $8 \%$ Glucose; at $\left.42^{\circ} \mathrm{C} ; \mathrm{pH}: 7.2\right)$

important concerning interferences by unselective ingredients of nutrition solutions. The bacteria growth exceeds the number of spores. It will be stopped only by a deficiency of nutrients. In this case the cells are transformed to spores, again. The incubation period is nearly indepen- dent of glucose concentration in nutrient solution. An increase of glucose concentration to $10 \%$ leads to a saturation effect of the bacteria sensor. This finding indicates a complete occupation of all receptor sites on the sensor coating by the analyte.

\section{Plastic Bioanalytes for Imprinting}

Natural bioanalytes can be considered as highly degradable materials which are not stable in the environment. Furthermore, as function of e.g. nutrition and temperature the properties of bacteria will be altered. Detection of a distinct type of species can be performed by plastic bioanalytes as shown in Fig. 9. These particles

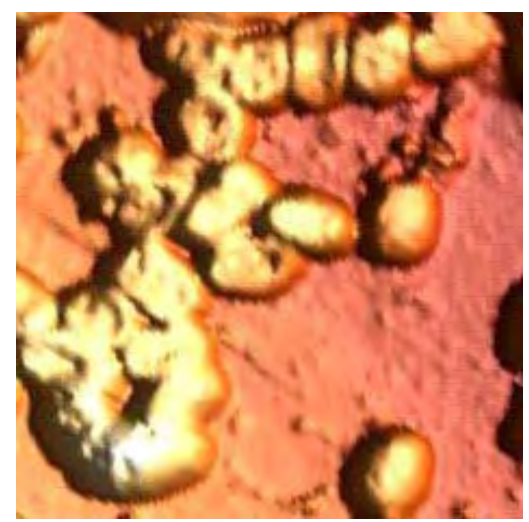

Fig. 9. Plastic bacteria on a flat surface as stamp for generating patterned QCM coatings

can be generated by a double imprinting process [3]. Furthermore, due to a high stability of these synthetic materials the stamps can be used for a long time.

\section{Conclusions}

Biomimetic imprinting strategies leads to more stable receptors as offered by nature. Its combination with sensor devices, especially masssensitive transducers, allows the selective detection of all types of bioanalytes from viruses, spores to cells in different sizes.

\section{References}

[1] O. Hayden, P.A. Lieberzeit, D. Blaas, F.L. Dickert, Artificial Antibodies for Bioanalyte Detection - Sensing Viruses and Proteins, Adv. Funct. Mater. 16, 1269-1278 (2006); DOI: 10.1002/adfm.200500626

[2] U. Latif, S. Can, O. Hayden, P. Grillberger, F.L. Dickert, Sauerbrey and anti-Sauerbrey behavioral studies in QCM sensors - Detection of bioanalytes, Sensors and Actuators B 176, 825830 (2013); dx.doi.org/10.1016/j.snb.2012.09.06

[3] M. Polreichova, U. Latif, F.L. Dickert, Functionalized Polymers as Receptors for Detection of Cells, Aust. J. Chem. 64, 1256-1260 (2011); /dx.doi.org/10.1071/CH11181 